\title{
Left-Turning Vehicle Trajectory Modeling and Guide Line Setting at the Intersection
}

\author{
Fulu Wei, ${ }^{1,2}$ Weiwei Guo, ${ }^{3}$ Xin Liu, ${ }^{4}$ Chunyan Liang, ${ }^{1}$ and Tianjun Feng ${ }^{1}$ \\ ${ }^{1}$ School of Science and Engineering on Communications, Jilin Jianzhu University, Changchun 130118, China \\ ${ }^{2}$ College of Transportation, Jilin University, Changchun 130022, China \\ ${ }^{3}$ Beijing Key Lab of Urban Intelligent Traffic Control Technology, North China University of Technology, Beijing 10014, China \\ ${ }^{4}$ Network Center, Jilin University, Changchun 130022, China
}

Correspondence should be addressed to Weiwei Guo; guoweiwei101@qq.com

Received 28 June 2014; Accepted 18 August 2014; Published 3 September 2014

Academic Editor: Yongjun Shen

Copyright (C) 2014 Fulu Wei et al. This is an open access article distributed under the Creative Commons Attribution License, which permits unrestricted use, distribution, and reproduction in any medium, provided the original work is properly cited.

\begin{abstract}
When left-turning vehicles are released from the multiple left-turning lanes at the signalized intersection, there will be conflicts among them, and the conflicts will affect the traffic operation and safety. In order to solve the problem, by extracting running trajectories of left-turning vehicles and analyzing distribution characteristics of trajectories, velocity changing characteristics, and flow changing characteristics, the left-turning vehicle's trajectory model was established. On the basis of the above research, taking an example of quadruple left-turning lanes, the idea of setting left-turning guide line at the intersection was proposed. Through instance verification, we could get the conclusion that the method of using left-turning guide line to control vehicles' turning process can effectively reduce traffic conflicts and delay and improve traffic efficiency.
\end{abstract}

\section{Introduction}

In most places of China, there is no lane guidance and constraint within the intersection, and many drivers want to enter the target lane as soon as possible under the psychological drive. When left-turning vehicles go through the stop line and enter the intersection, many turning vehicles often change driving route randomly. The phenomenon of vehicles' scattering randomly within the intersection causes serious conflicts among vehicles and makes vehicles not able to run smoothly. It reduces traffic efficiency and safety. Thus, by analyzing and researching the trajectories of left-turning vehicles at the intersection, the method of setting guide line was proposed. The method of guiding vehicles turning in line appears to be necessary and feasible for improving the level of left-turning vehicles' efficiency and safety at the intersection. It has a certain theoretical and practical value.

In the recent years, in order to reduce the delay of left-turning vehicles at the intersection and improve traffic operational performance, many scholars have done some studies about it from different angles. For example, Ma and
Yang [1] studied the crowded and noncrowded intersection's left-turning group coordinated design approach, considering both phase coordination and spatial coordination, and established optimization model in order to minimize the total delay; Kikuchi et al. [2] studied the effect of dual left-turning lanes' lengths on operating. Shen [3] studied the relationship between vehicle's average delay and merging section length. And she proposed a method to determine the minimum merging section lengths for triple left-turning lanes whose downstream lanes was less than the entrance lanes. Eyler [4] studied the problem of triple left-turning lanes which were used at large intersections and caused high crash rates and traffic delays. And the arterial interchange concept was proposed. Wu et al. [5] extracted the left-turning vehicles trajectories data using the video method and proposed a translating method of the image coordinates and the world coordinates. Kyte et al. [6] proposed the IQA method for estimating the left-turning delay. Qi et al. [7] used different models to estimate the left-turning queue length, and the results of a simulation-based method were compared with others. Yao [8] Yao and Zhang [9] studied the signal timing 
and lane space design of short left-turning lanes at the isolated intersection or the two adjacent intersections; Liu et al. [10] studied the drivers' choice behavior on the left-turning lanes and pointed out the main factors on outside left-turning lanes selection. Moussa et al. [11] used augmented reality vehicle (ARV) system to study the maneuver of left-turning traffic. A lot of virtual objects, such as pedestrians, vehicles, and others, could be seen while driving in a real traffic environment. By the experiment result, the "ARV" system could improve drivers' performance effectively. Alhajyaseen et al. [12] recorded individual vehicle paths, using Euler spiral curve and circle curve and considering intersection geometry, different vehicle type, and speed. Alhajyaseen et al. noted that vehicle paths were very important in analyzing driver maneuver. Li et al. [13] studied the impact factors of major leftturning delay on signal setting. Sando and Moses [14] investigated 15 triple left-turning lanes intersections and studied the influence of geometric factors on the operation of left-turning traffic. Wu et al. [15] optimized left-turning phases to reduce delay. These research results have some effects on reducing left-turning delay, conflicts, and so on. However, when leftturning vehicles go through the stop line and enter the intersection, the problem of vehicles interfering with each other has not gotten a good solution. Vehicles randomly change their lines, which affects the left-turning vehicles' safety level and operational performance in their turning process. Wang et al. [16] proposed novel cell transmission model to simulate the oversaturated traffic conditions of left-turning blockages. Cooner et al. [17] put forward the preliminary thought of using guide lines for triple left turns and dual right turns in Texas. Bie et al. [18-20] studied the prediction of bus arrival and other relevant research contents, which could be used in the formulation of signal control strategy of multiple left-turning lanes intersection in the future.

On the basis of existing studies, the paper summarized predecessors and studied the vehicles' turning trajectories inside the intersection deeply. From the perspective of both reducing traffic conflicts and improving efficiency, we want to adopt some measures to guide turning flow and restrain vehicles' lane changing.

\section{The Left-Turning Trajectory Extraction}

On the basis of summarizing the existing literature, the current research adopts the method of video detection to extract vehicles' turning trajectories and studies the characteristics of turning vehicles. The current study intends to build a turning trajectory model which could be used to show the characteristics of turning vehicles and exhibit the turning process of vehicle. Therefore, the process of conflicts of turning vehicles could be illustrated vividly and further studied. Because of the existence of serious conflicts among multiple left-turn vehicles, the suggestion of setting guide line at the multiple left-turn lanes intersection is proposed. The logical framework is shown in Figure 1.

2.1. Extraction of Vehicle Center and Correction of Camera Lens Distortion. The trajectories of the vehicles can effectively reflect the changes of their spatial locations, velocity,

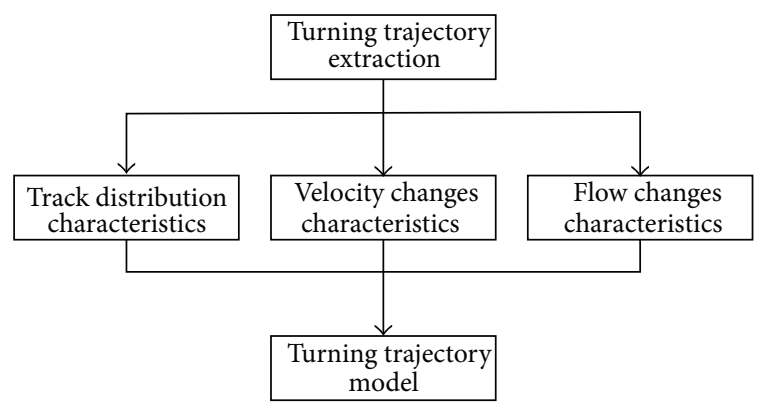

FIgURE 1: The establishment process of the trajectory model.

acceleration, the conditions of traffic flow, and the density of vehicles at a particular time state. And the trajectories could provide experimental data for vehicles interaction model, such as acceptable gap model, following model, and lane changing model. In this paper, video detection measures are used to achieve real-time trajectories of the left-turning vehicles inside the intersection. Firstly, the centers of the turning vehicles inside the intersection are extracted by using complex extraction algorithm. And then their centers are tracked, the center position coordinates at various times are recorded. Considering the camera's lens distortion, its center is corrected. At last, the corrected image coordinates are converted into world coordinates, and the trajectories of the vehicles are gotten. The center of turning vehicle is calculated by the following equations:

$$
\begin{aligned}
& G(x)=\frac{\sum_{x_{c} \in G_{s}} x_{c}}{\sum_{x_{c} \in G_{s}} M}, \\
& G(y)=\frac{\sum_{y_{c} \in G_{s}} y_{c}}{\sum_{y_{c} \in G_{s}} M},
\end{aligned}
$$

where $G_{s}$ is contour information of the vehicle and $M$ represents the number of pixels included in $G_{s}$.

The image area is divided into the $m \times n$ rectangular region, and the center coordinate of the image area is $c^{\prime}\left(x^{\prime}, y^{\prime}\right)$, through the polygon linear scan that could obtain the center coordinate as $c^{\prime \prime}\left(x^{\prime \prime}, y^{\prime \prime}\right)$. Let the extracted center coordinate of the foreground image be $\left(x_{z}, y_{z}\right)$; the establishment calculation model for correcting distortion is calculated as

$$
\begin{aligned}
& x_{z}=\left(\frac{\left|x_{z}^{\prime}-x_{a}\right|}{\left|x_{c}-x_{a}\right|}+\sum_{i=1}^{n}\left(\frac{n}{2}-i\right) \beta_{x}\right) w \\
& y_{z}=\left(\frac{\left|y_{z}^{\prime}-y_{b}\right|}{\left|y_{d}-y_{b}\right|}+\sum_{j=1}^{m}\left(\frac{m}{2}-j\right) \beta_{y}\right) l
\end{aligned}
$$

where $x_{a}, x_{c}, y_{b}$, and $y_{d}$ are intersection coordinates of polygon scan line and the 4 sides of target area. $w, l$ are defined as the center line of the image in the $x$-direction and 


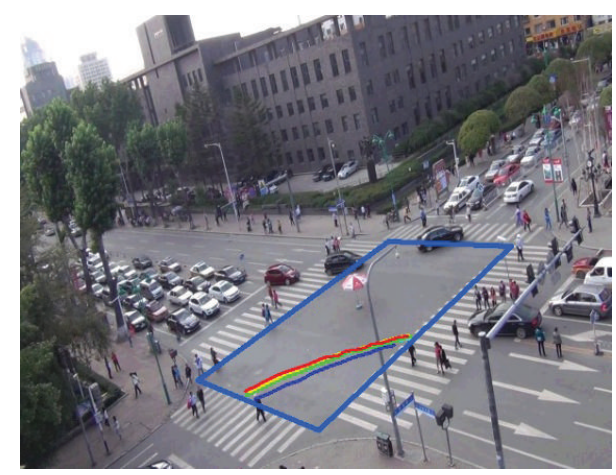

FIGURE 2: Extraction of left-turning vehicles' trajectories schematic diagram.

$y$-direction length and $\beta_{x}, \beta_{y}$ are offset scale factors, which are calculated as

$$
\begin{aligned}
& \beta_{x}=\frac{8\left(x^{\prime}-x^{\prime \prime}\right)}{w n(2+n)}, \\
& \beta_{y}=\frac{8\left(y^{\prime}-y^{\prime \prime}\right)}{\operatorname{lm}(2+m)} .
\end{aligned}
$$

Using the above method, left-turning vehicles' trajectories at the intersection could be extracted. Taking the intersection of Tongzhi Street and Ziyou Road in Changchun City as an example, we extracted left-turning vehicles' trajectories from east to south and one of the images is shown in Figure 2.

\subsection{Extraction of Displacement of Vehicle and Estimation of} Velocity. Based on the extraction of the centers of vehicles and camera lens distortion correction, the improved method of Kalman filtering is used to track the centers of turning vehicles and record the changing of the centers' position, which could determine the coordinates of the centers of the vehicles in each time. Since the change time of each frame image is $0.04 \mathrm{~s}$, in this very short time the changes of vehicle's center position are very small. Thus, every five frames time is $0.2 \mathrm{~s}$, which are selected as a time interval for studying in the calculation process. Therefore, displacement of vehicle in the time interval can be expressed as

$$
\text { Disp }=\operatorname{sqrt}\left(\left(x_{k+1}-x_{k}\right)^{2}+\left(y_{k+1}-y_{k}\right)^{2}\right) .
$$

The average speed of the vehicles within this time interval can be expressed as

$$
v=\frac{\text { Disp }}{\text { Nof } * t},
$$

where Nof is the number of image frames which is contained in the driving displacement corresponding to the time interval and $t$ is the time of each frame image, which is $0.04 \mathrm{~s}$ in the paper.

By extracting the displacement of vehicle in the turning process, recording vehicles' turning time, and the number of turning vehicles, the average velocity of turning vehicles can be calculated as

$$
\bar{v}=\frac{\text { veh. } n * \sum_{i=1}^{m} \operatorname{Disp}_{i}}{\sum_{j=1}^{\text {veh.n}} t_{j}} .
$$

The calculation of average turning velocity of the vehicles can be used to measure the traveling efficiency of vehicles under different conditions of setting guide line or without setting diversion line.

\section{Left-Turning Vehicle Trajectory Model}

3.1. Turning Characteristics of Left-Turning Vehicles. Based on a long-term observation and research of types of intersections' vehicles, some characteristics of left-turning vehicles are found which are shown at the process of turning, through the extraction, and summarizing the characteristics of the turning vehicles, in-depth understanding of the turning process. In order to establish the model of left-turning vehicles' objectively and effectively, some basic preparations are provided. Left-turning vehicles' characteristics will be elaborated from three aspects as follows.

(1) Distribution of Trajectories. In the same period of a green phase time, from the beginning of green to the end, the vehicles' tightly wrapped layer by layer from outside to inside and from sparse to dense, which could form an envelope surface. After the left-turning vehicles' trajectories of 120 cycles were extracted and researched, the same conclusion could be got. As shown in Figure 3, with the order of the queue turning vehicles' trajectories going through the intersection is extracted, at a left-turning green phase time. In order to easily distinguish the trajectories of different vehicles, different colors are used to mark different order trajectories, such as red, yellow, and blue. Trajectory distribution characteristics could provide a reference for the choice of guide line setting area.

(2) Turning Velocity Variation Characteristics. By tracking and monitoring the velocity of left-turning vehicles, we could discover that when left-turning vehicles change their lanes during their turning progress, the rear vehicle's velocity will show obvious pause or slow down. On the contrary, if all the left-turning vehicles do not change their lanes during their turning progress, the fluctuation of velocity will be small and the traffic release will be stable and efficient. So it is necessary to guide the vehicles' turning progress and reduce the lane changing behavior. The velocity changes reflect the severity of conflicts between left-turning vehicles and drivers are forced to take measures to ensure personal security. On the other side, the extraction of velocity could be used to calculate the vehicles' turning angle in the process of trajectory modeling.

(3) Flow Distribution Characteristics of Different Cross Section. From the intersection's entrance, diagonal, export three sections, and the distribution characteristics of flow with time are studied when a left-turning flow entered the intersection. From the change of each section in flow over time diagram, 


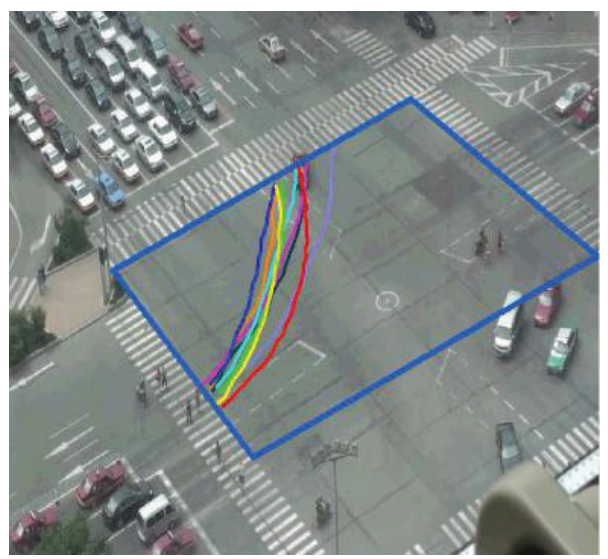

(a) Intersection of R\&Z

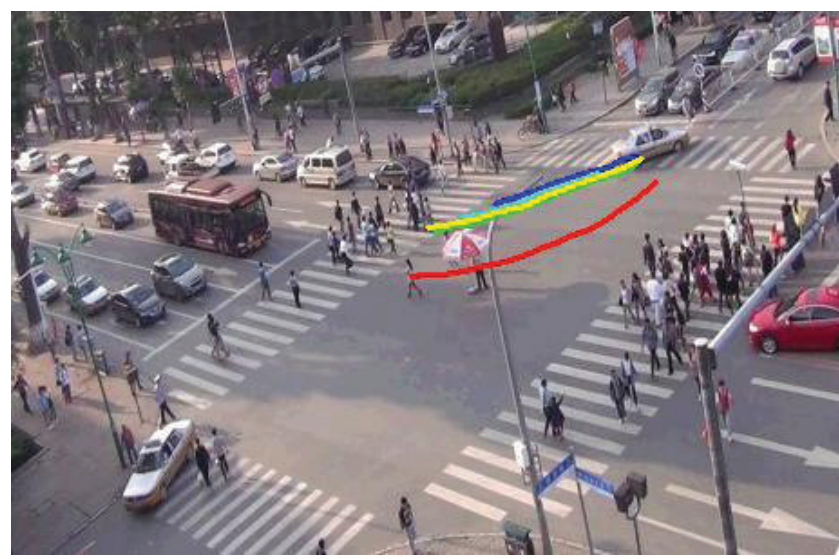

(b) Intersection of Z\&T

FIGURE 3: Vehicles' trajectories inside a green phase.

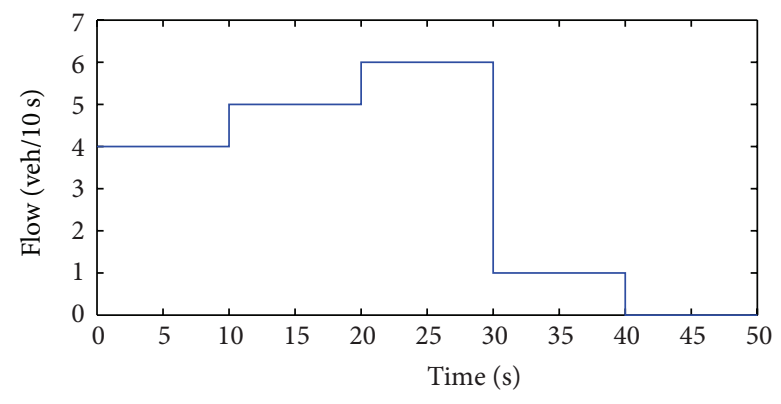

(a) The entrance

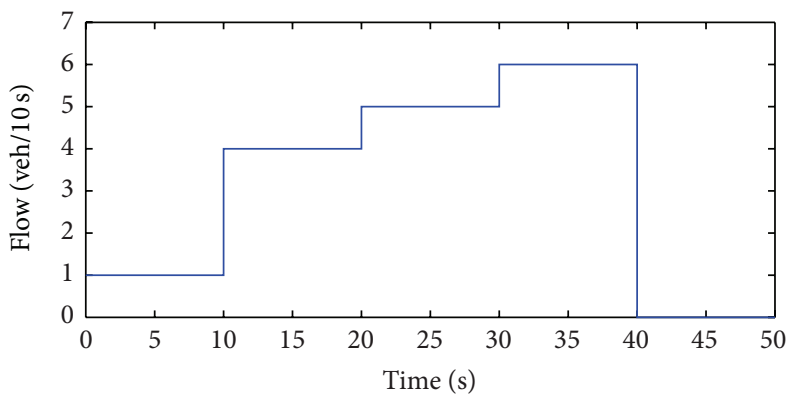

(b) The diagonal

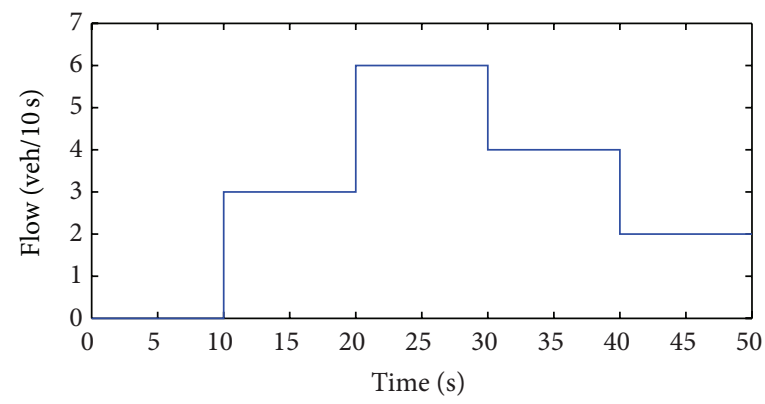

(c) The export

FIgURE 4: The relationship between flow and time in different sections.

the discrete characteristics can be understood in the vehicles' turning process and the flow changes may also be reflected in the condition of the vehicles' lateral conflicts. Make a left-turning green phase at the intersection, for example, which demonstrates the changes in flow over time in different cross-section, as shown in Figure 4. When there are conflicts between vehicles and traffic block, the flow of every section will change accordingly.

3.2. Development of the Trajectory Model. During the process of turning, the vehicles' position continuously changes with the change of angles, velocities, and displacements. Different vehicles' turning trajectories may have some types of difference. But at the same intersection whose channelization scheme is fixed, the trajectories of the turning vehicles show some regularity. And according to the principle of optimization, the trajectories of the turning vehicles always tend to a reasonable path. The left-turning trajectory diagram can be seen as shown in Figure 5.

According to the knowledge of probability and statistical analysis, a large number of turning vehicles' trajectories are extracted and counted. Combined with the left-turning characteristics, such as trajectory distribution, velocity distribution characteristics, the trajectories whose distribution is abnormal are removed and the trajectories which are produced by the big velocity fluctuation vehicles should also be removed. Therefore, the vehicle trajectory samples which are formed in the normal turning conditions are obtained. 


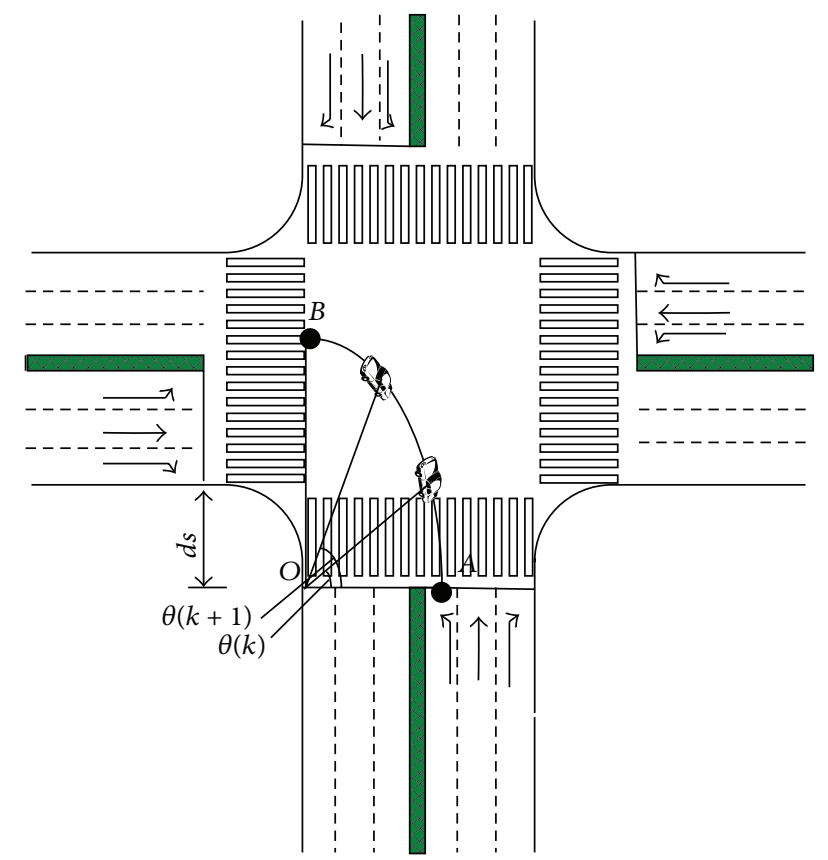

FIGURE 5: Left-turning vehicle’s trajectory diagram.

Covered with these trajectory samples, the region formed a geometric figure. And then, the geometric figure's center line is extracted from the entrance to the exit lane direction. After the center line is processed smoothly, a reasonable path for left turn vehicles is shown. Establish model for the reasonable path trajectory and take the intersection in Figure 5 as an example; the reasonable path of the left-turning vehicles is shown as $A B$ arc. Let the number of lanes in east-west direction at the intersection be EW.In, let lane width be EW.lw, and let green belt be EW.grw; and the number of lanes in south-north direction is SN.ln, lane width is SN.lw, and green belt is SN.grw. Safety lateral clearance is $\alpha_{1}$ and $\alpha_{2}$. Point $O$ was regarded as the turning center, which is located at the lower left corner of the intersection. ds is the vertical distance from the outermost lane boundary of the west entrance to the point $O . O A, O B$, which are the turning radius of the arc start and end, are defined as $R_{s l}$ and $R_{w l}$ which are calculated as

$$
\begin{aligned}
R_{s l} & =\mathrm{SN} \cdot \mathrm{lw} \times(\mathrm{SN} \cdot \ln +0.5)+\mathrm{SN} \cdot \mathrm{grw}+\alpha_{1}, \\
R_{w l} & =\mathrm{EW} \cdot \mathrm{lw} \times(\mathrm{EW} \cdot \ln +0.5)+\mathrm{EW} \cdot \mathrm{grw}+\mathrm{ds}+\alpha_{2} .
\end{aligned}
$$

When the left-turning vehicle position changes, the turning radius of the arc may be expressed as

$$
R_{l}=\operatorname{Sqrt}\left(\left(R_{s l} \times \operatorname{Sin}(\theta(k))\right)^{2}+\left(R_{w l} \times \operatorname{Cos}(\theta(k))\right)^{2}\right) .
$$

The start turning angle is $\theta(k)=0$ and the start point is $\operatorname{Arc} A=A$.

Let vehpos. $x(k)$ be vehicle's $X$ coordinates at $K$ moment; let vehpos. $y(k)$ be vehicle's $y$ coordinates at $K$ moment. The position of the left-turning vehicle is decided by the start

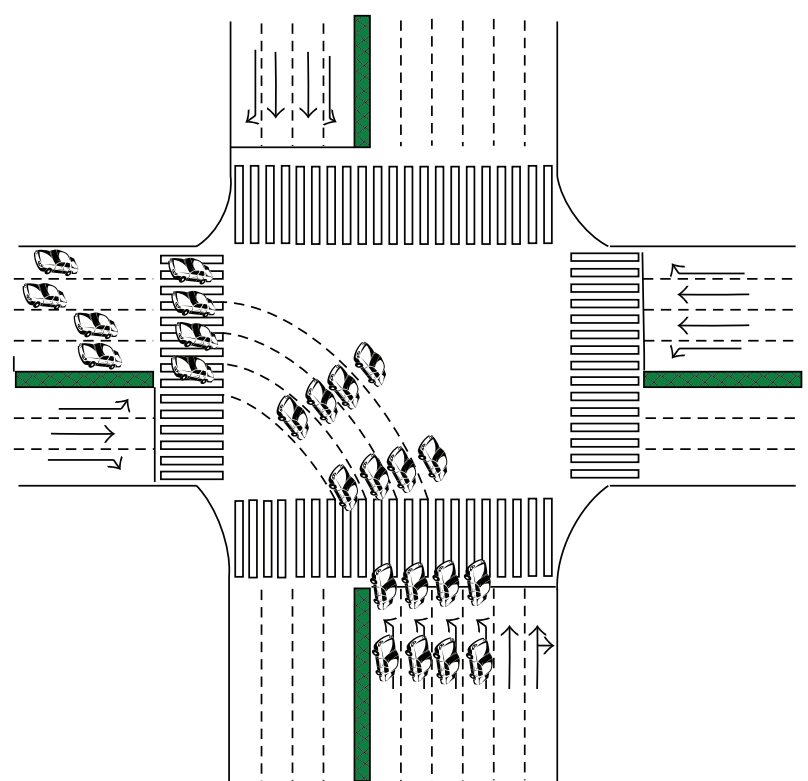

Figure 6: Left-turning guide line setting.

and end turning radius, the start and end positions, speed, vehicle's locations, and turning angles, which is calculated as

$$
\begin{aligned}
\operatorname{vehpos} x(k+1) & =\operatorname{Arc} A \cdot x+R_{l} * \sin \theta(k+1), \\
\text { vehpos. } y(k+1) & =R_{l} * \cos \theta(k+1), \\
\theta(k+1) & =\theta(k)+\frac{\text { vehSpeed }}{R_{l}},
\end{aligned}
$$

where vehSpeed is the average velocity of vehicles from the $k$ to $k+1$ moment, which can be calculated by extracting data of the trajectory model. The trajectory model could be used not only in the single left-turning lane but also in the multiple left-turning lanes.

\section{Left-Turning Guide Line Setting at the Intersection}

Based on the extraction of the left-turning trajectories and modeling, we can learn that conflicts among vehicles, as a universal phenomenon, exist if there are no guide lines at the intersection. Thus, some restraint measures should be taken. And setting guide lines for multiple left-turning lanes at the intersection is proposed which could be used to guide the traffic. Take a quadruple left-turning lanes intersection, as an example, which is shown in Figure 6.

Left-turning guide lines are set between the inlet and outlet sidewalks inside the intersection, which is mainly from the following two considerations. On one hand, the end of the guide line ends at the exit of left-turning of the sidewalk closed beta, which can give left-turning vehicles enough freedom in choosing the exit road. Driver could choose a reasonable road timely to leave the intersection fast, according to the driving conditions and space situation in 
TABLE 1: The calculation results.

\begin{tabular}{lcc}
\hline The same left lane & $\begin{array}{c}\text { Setting guide } \\
\text { lines }\end{array}$ & $\begin{array}{c}\text { Without guide } \\
\text { lines }\end{array}$ \\
\hline Average driving delay $(\mathrm{s})$ & 0.1 & 0.21 \\
Average driving velocity $(\mathrm{km} / \mathrm{h})$ & 32.6 & 15.8 \\
\hline
\end{tabular}

front of the vehicle. On the other hand, in giving the leftturning vehicles' freedom to choose exit lane to turn, at the same time, a certain limit is generated on left-turning path at the process of turning. The setting of guide line could reduce conflicts between left-turning vehicles, because of changing lanes randomly during the progress of turning left, and ensure the turning vehicles driving fast and efficiently in the guiding of diversion line.

Vehicles of the left-turning lanes go through the stop line and crosswalk into the intersection; because of the in front guide lines' guiding and binding effect, the left-turning vehicles' flow generally will not appear as dispersed phenomenon. So the starting point of diversion line can be disposed inside the crosswalk. When any vehicle drives to the end of the guide line, the driver will make a decision to choose a suitable path, according to the destination location, driving conditions goals, and the running state of target lane in front of the vehicle. The diversion line is set in this way, which could make left-turning vehicles run orderly and quickly under the constraints, without losing the flexibility and freedom.

The intersection of Figure 6 has quadruple left-turning lanes. Before the setting of guide line, and conflicts between vehicles will happen in the progress of release which will affect the traffic efficiency. After the guide lines are set, the majority of vehicles will follow the guide line, and the quantity of conflicts will be reduced.

After the left-turning guide lines are set, vehicles will intent to follow the guiding of guide lines. From both the perspective of the binding of regulations and the view of psychology, the majority of drivers will travel along the route of guide line and form a very neat turning queue. The phenomenon of vehicles changing their route randomly can be greatly reduced, the state of vehicles disorderly distributed inside the intersection could also be improved, and the conflicts between left-turning vehicles will be reduced. Leftturning traffic flow will accelerate in the process of traveling from entering the intersection to exit the intersection, which will improve the efficiency of the turning vehicle traffic.

\section{Instance Discussion}

A long-term investigation has been done about the intersection of Yatai Street and Nanhu Road which has quadruple left-turning lanes in Changchun China, as shown in Figure 7. The average traffic delay and driving velocity were contrasted, between the two states of lane changing and without lane changing. Here, we assumed that the state of without lane changing was just like the state of setting guide line. And the calculation results are as shown in Table 1.

From Table 1, we can see that the delay of no lane changing or setting guide lines is lower than that without setting guide

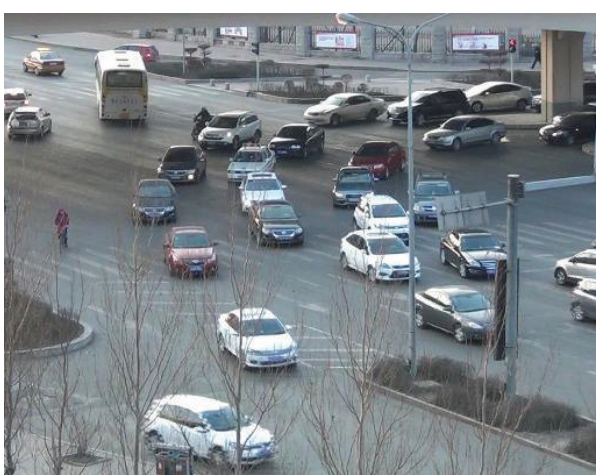

FIGURE 7: The quadruple left-turning lanes intersection.

lines and the average driving velocity of setting guide lines is higher than that without setting guide lines. So guide line's setting can reduce left-turning vehicles' delay at the intersection and improve traffic efficiency.

\section{Conclusion}

This paper applies the method of video detection to realize real-time extraction and tracking of turning vehicles' trajectories at the intersection. The distribution characteristics of trajectories, the changing characteristics of velocity, and the changing characteristics flow are analyzed in depth. And the trajectory model of left-turning vehicles is established. Considering the vehicles' turning characteristics and the trajectory model, the method of setting guide line is proposed. The intersection with quadruple left-turning lanes is demonstrated as an example. Finally, the intersection of Yatai Street and Nanhu Road is used to verify the effect of setting guide lines at the intersection. The result shows that, under the guiding of guide line, vehicles' mutual interference could be reduced effectively. Thus, the study provides a theoretical basis for the traffic channelization of intersection and has a good application prospect.

For further issues, we will consider how to set the guide line in other states. For example, when the number of leftturning entrance lanes is less than the number of exit lanes, we should adopt which method to set the guide line. And we will also study the conflict mechanism among vehicles at the intersection.

\section{Conflict of Interests}

The authors declare that there is no conflict of interests regarding the publication of this paper.

\section{Acknowledgments}

This work is partly supported by the National Natural Science Foundation of China (nos. 51278220, 61104168) and China Scholarship Council. The authors thank the anonymous reviewers for their valuable input and suggestions. 


\section{References}

[1] W. Ma and X. Yang, "Coordination design of left movements of signalized intersections group," Journal of Tongji University: Natural Science Edition, vol. 36, no. 11, pp. 1507-1511, 2008.

[2] S. Kikuchi, M. Kii, and P. Chakroborty, "Lengths of double or dual left-turn lanes," Transportation Research Record, vol. 1881, pp. 72-78, 2004.

[3] Q. Shen, "Minimum merging section lengths for triple left-turn lanes with downstream lane reductions," ITE Journal, vol. 71, no. 3, pp. 40-45, 2001.

[4] D. R. Eyler, "Arterial interchange," Transportation Research Record, vol. 1912, pp. 65-71, 2005.

[5] J. Wu, C. Xue, Z. Zhao, and B. Liu, "Approach towards leftturning vehicles trajectory at the intersection based on video technology," in Proceedings of the International Conference on Communication, Electronics and Automation Engineering, vol. 181 of Advances in Intelligent Systems and Computing, pp. 1-7, 2013.

[6] M. Kyte, A. Abdel-Rahim, M. Dixon, J.-M. Li, and D. Strong, "Validating the incremental queue accumulation method for left-turn delay estimation," Transportation Research Record, vol. 2130, pp. 42-51, 2009.

[7] Y. G. Qi, L. Guo, L. Yu, and H. Teng, "Estimation of design lengths of left-turn lanes," Journal of Transportation Engineering, vol. 138, no. 3, pp. 274-283, 2012.

[8] R. Yao, "Sensitivity analysis of optimization models for two adjacent intersections with correlated short left-turning lanes," Transport, vol. 28, no. 3, pp. 256-269, 2013.

[9] R. Yao and H. M. Zhang, "Optimal allocation of lane space and green splits of isolated signalized intersections with short leftturn lanes," Journal of Transportation Engineering, vol. 139, no. 7, pp. 667-677, 2013.

[10] P. Liu, C. Xu, and W. Wang, "Identifying factors affecting drivers' selection of unconventional outside left-turning lanes at signallised intersections," IET Intelligent Transport System, vol. 7, no. 4, pp. 396-403, 2013.

[11] G. Moussa, E. Radwan, and K. Hussain, "Augmented reality vehicle system: left-turn maneuver study," Transportation Research Part C: Emerging Technologies, vol. 21, no. 1, pp. 1-16, 2012.

[12] W. K. M. Alhajyaseen, M. Asano, H. Nakamura, and D. M. Tan, "Stochastic approach for modeling the effects of intersection geometry on turning vehicle paths," Transportation Research C, vol. 32, pp. 179-192, 2013.

[13] R. Li, W. Li, and F. Qiu, "Signal setting critical volume for two-way stop-controlled intersection based on major left-turn delay," Journal of Southeast University (Natural Science Edition), vol. 42, no. 3, pp. 551-554, 2012.

[14] T. Sando and R. Moses, "Influence of intersection geometrics on the operation of triple left-turn lanes," Journal of Transportation Engineering, vol. 135, no. 5, pp. 253-259, 2009.

[15] W. Wu, W. Ma, and X. Yang, "Integrated optimization method for left-turn phases of adjacent junctions," Journal of Tongji University, vol. 41, no. 1, pp. 66-71, 2013.

[16] P. Wang, S. L. Jones, S. Gurupackiam, and L. Wang, "Novel cell transmission model-based simulations of left-turning blockages inside intersections," Transportation Research Record, vol. 2390, pp. 60-67, 2013.

[17] S. A. Cooner, S. E. Ranft, Y. K. Rathod et al., "Development of guidelines for triple left and dual right-turn lanes," Project performed in cooperation with the Texas Department of Transportation and Federal Highway Administration, 2011.

[18] Y. Bie, D. Wang, and H. Qi, "Prediction model of bus arrival time at signalized intersection using GPS data," Journal of Transportation Engineering, vol. 138, no. 1, pp. 12-20, 2012.

[19] Y. Bie, Z. Liu, D. Ma, and D. Wang, "Calibration of platoon dispersion parameter considering the impact of the number of lanes," Journal of Transportation Engineering, vol. 139, no. 2, pp. 200-207, 2013.

[20] Y. Bie, D. Wang, and X. Qu, "Modelling correlation degree between two adjacent signalised intersections for dynamic subarea partition," IET Intelligent Transport Systems, vol. 7, no. 1, pp. $28-35,2013$. 


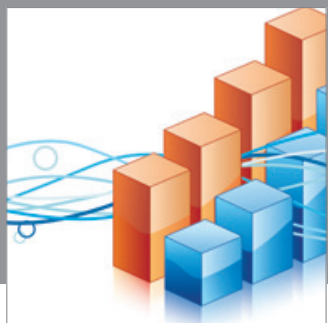

Advances in

Operations Research

mansans

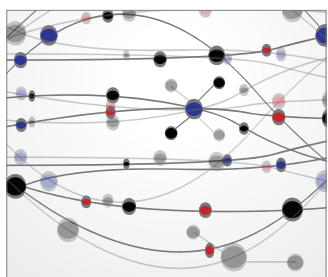

The Scientific World Journal
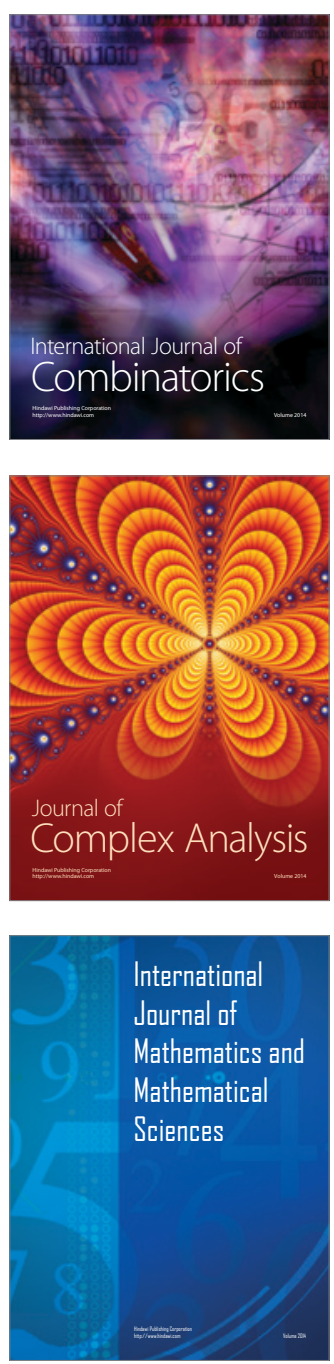
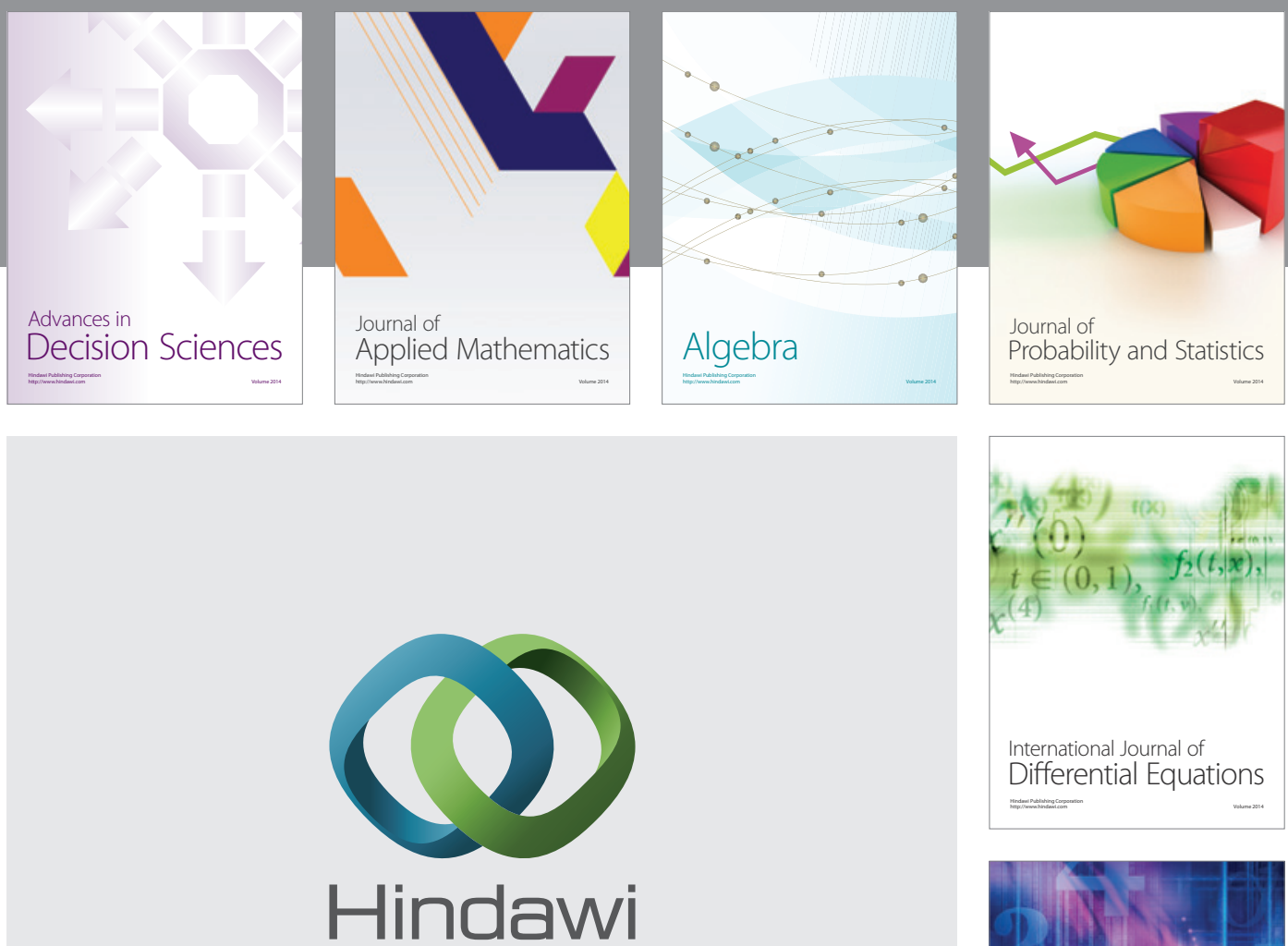

Submit your manuscripts at http://www.hindawi.com
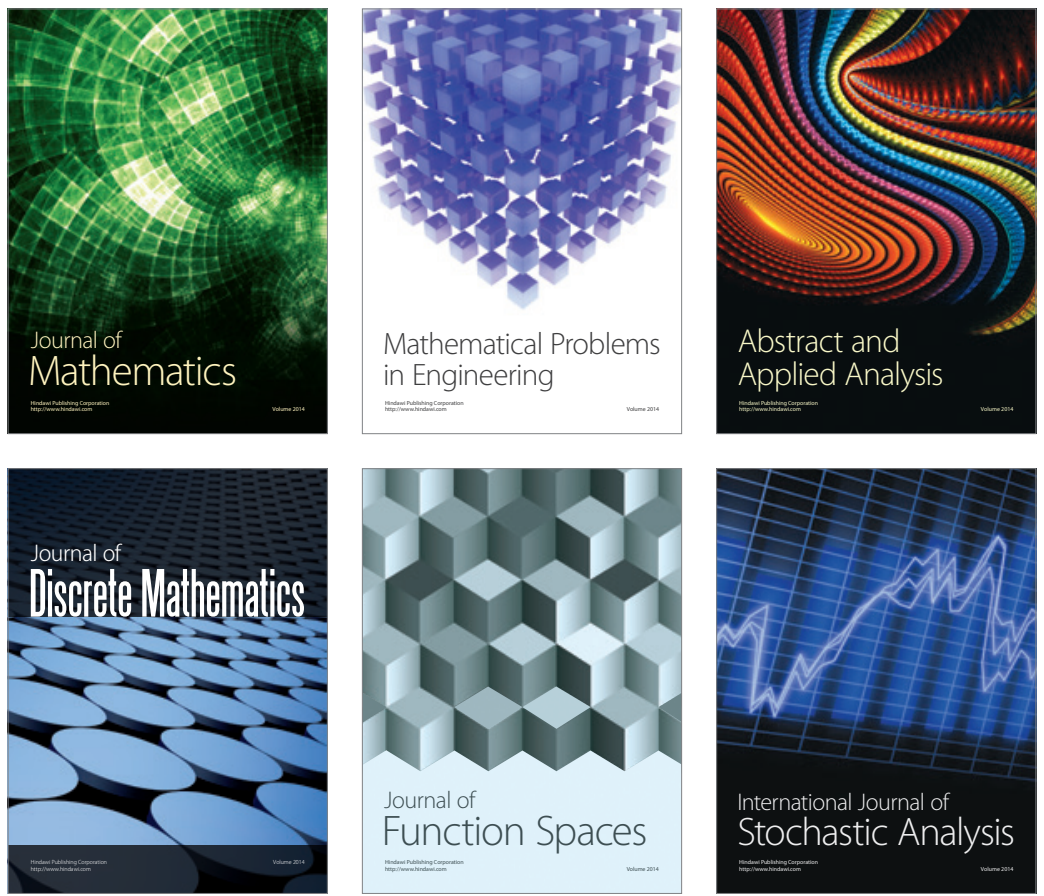

Journal of

Function Spaces

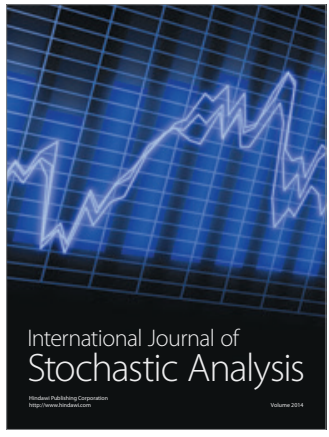

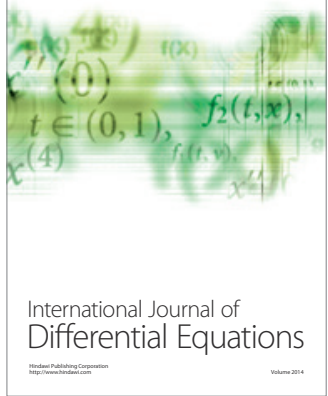
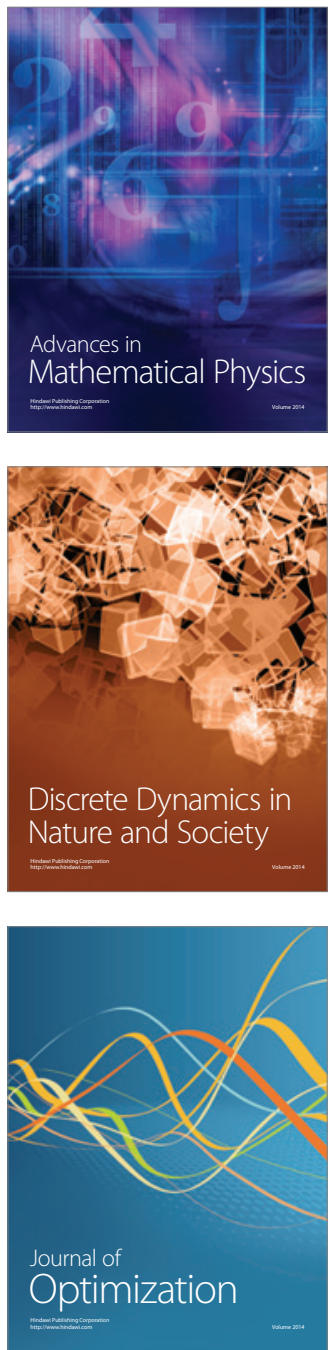